\title{
Heat-Bath Cooling of Spins in Two Amino Acids
}

\author{
Y. Elias ${ }^{\mathrm{a}, *}$, H. Gilboa ${ }^{\mathrm{a}}$, T. Mor ${ }^{\mathrm{b}}$, Y. Weinstein ${ }^{\mathrm{c}}$ \\ ${ }^{a}$ Schulich Faculty of Chemistry, Technion 32000, Haifa, Israel \\ ${ }^{b}$ Department of Computer Science, Technion 32000, Haifa, Israel \\ ${ }^{c}$ Department of Physics, Technion 32000, Haifa, Israel
}

\begin{abstract}
-Heat-bath cooling is a component of practicable algorithmic cooling of spins, an approach which might be useful for in vivo ${ }^{13} \mathrm{C}$ spectroscopy, in particular for prolonged metabolic processes where substrates that are hyperpolarized ex-vivo are not effective. We applied heat-bath cooling to $1,2-{ }^{13} \mathrm{C}_{2}$-amino acids, using the $\alpha$ protons to shift entropy from selected carbons to the environment. For glutamate and glycine, both carbons were cooled by about 2.5 -fold, and in other experiments the polarization of $\mathrm{C} 1$ nearly doubled while all other spins had equilibrium polarization, indicating reduction in total entropy. The effect of adding Magnevist ${ }^{\circledR}$, a gadolinium contrast agent, on heat-bath cooling of glutamate was investigated.
\end{abstract}

\section{Introduction}

Heat-bath cooling [1] is a key building-block of algorithmic cooling $(A C)$, a recent spin-cooling approach originally proposed for purifying qubits in NMR quantum computers [2, 3]. AC intersperses heat-bath cooling (which combines polarization transfer and fast repolarization) with reversible polarization compression. By itself, polarization compression [4, 5] is limited due to the entropy bound (also called Shannon's bound) [6], and further limited by the unitary bound on spin dynamics [4]. However, heatbath cooling allows AC to cool spins (spin-half nuclei) far beyond these bounds [2, $3,2,7,8,9,10]$. AC, as well as its components, heat-bath cooling and polarization compres'sion, may enhance in vivo ${ }^{13} \mathrm{C}$ magnetic resonance spectroscopy (13C-MRS), which enables dynamic analysis of metabolic processes in living organisms [11, 12].

Heat-bath cooling achieves controlled entropy extraction from suitable spin systems [1] by applying one or more steps of selective-reset. Each selective-reset cools a 'selected slow-relaxing spin (computation spin) by polarization transfer (PT) from a more polarized reset spin, and this PT is followed by a delay. We call this idle step (the delay) a reset step. The reset spin repolarizes rapidly during that reset step so that the spin system is cooled; the reset spin may be reused via an iterative (or recursive) algorithm [2, 3]. For a pair of computation and reset spins, respectively $c$ and $r$, we denote here by $\mathcal{R}(c, r)$ the ratio between the thermalization time of the computation spin, $T_{1}(\mathrm{c})$, and the characteristic repolarization time of the reset spin (for liquid NMR, it is the thermalization time,

*Presented at the International Society for Magnetic Resonance (ISMAR) in Florence, 2010.

**Corresponding author. Fax: +972 48293900.

Email address: ye1@technion.ac.il (Y. Elias)
$\left.T_{1}(\mathrm{r})\right)$. A large ratio, $\mathcal{R}(c, r) \gg 1$, is required to preserve most of the enhanced polarization of the computation spin during reset steps. In addition, strong scalar J-coupling (or for solids dipolar coupling) is required, $J \gg 1 / T_{2}$, such that loss of polarization by decoherence during PT would be minimized.

$\mathrm{AC}$ produces spin-cooling using heat-bath cooling steps, wherein the spin entropy is transiently reduced (the spins are cooled) even though no physical cooling is applied to the system. Practicable cooling algorithms that yield moderate cooling already for small spin systems were suggested [3, 8], and improved algorithms were recently derived [10]. Heat-bath cooling, by itself, can already cool the entire spin system, bypassing the entropy bound, if, at equilibrium, the reset spin polarization, $\varepsilon(r)$, is higher than the polarization of the computation spins, $\varepsilon(c)$ [3].

For larger spin systems, AC can theoretically generate hyperpolarized spins, for which the polarization is increased by several orders of magnitude [2, 3, 7, 8, 9, 10]. However, such extensive cooling faces significant obstacles and requires many selective-resets and is not expected in the near future. Other spin-cooling methods that produce hyperpolarization include dynamic nuclear polarization in the solid state followed by rapid thawing (dissolution DNP) 13], parahydrogen induced polarization ( $\mathrm{PH}-$ IP) [14], and optical pumping of noble gases 15]. For small spin systems, AC is applicable with current technology, and has two important advantages over dissolution DNP, the most generally applicable hyperpolarization method: both $\mathrm{AC}$ and heat-bath cooling require no special apparatus and may therefore be applied directly in vivo, in particular to enhance 13C-MRS of slow metabolism in the brain; Furthermore, the polarization enhancement may be applied/replenished in vivo after several hours.

Significant entropy reduction was already demonstrated 
experimentally 1]. That heat-bath cooling experiment used ${ }^{13} \mathrm{C}_{2}$-trichloroethylene (TCE) in chloroform solution (with $\varepsilon(r) \sim 4 \varepsilon(c)$ ), a simple model system comprising three spins - two carbons and one proton, where C1 is the quaternary carbon and $\mathrm{C} 2$ is bound to the proton. A paramagnetic reagent, $\mathrm{Cr}(\mathrm{acac})_{3}$, improved both $T_{1}$ ratios [16], such that $\mathcal{R}(\mathrm{C} 1, \mathrm{H}) \sim 10$ and $\mathcal{R}(\mathrm{C} 2, \mathrm{H}) \sim 5$. The entire spin system was cooled beyond the entropy bound by a dual selective-reset, using the four-step pulse sequence POTENT (POlarization Transfer by ENvironment Thermalization) [1]:

1. $\mathrm{PT} \# 1 . \mathrm{H} \rightarrow{ }^{13} \mathrm{C} \rightarrow{ }^{13} \mathrm{C}(H C C)$ relay implemented by two refocused INEPT sequences [17, 18] using non-selective ("hard") pulses.

2. Reset \#1. A significant delay of $\sim 2 T_{1}(\mathrm{H})$.

3. PT \#2. Refocused INEPT from the partly repolarized proton to $\mathrm{C} 2$.

4. Reset \#2. A significant delay of $\sim 3 T_{1}(\mathrm{H})$.

After POTENT, both ${ }^{13} \mathrm{C}$ were cooled nearly two-fold, while the proton regained most of its equilibrium polarization, such that the entire spin system was cooled. In the submitted version of [1], another heat-bath cooling experiment is described where only steps $1-3$ above are performed, such that both carbons are cooled, each by a factor greater than two.

We chose to work here with glycine and glutamate, which are both neurotransmitters [19]; Glutamate has the major excitatory role in the human brain, while glycine assumes an inhibitory function in specialized neurons [19] and also acts as a cofactor for excitatory neurotransmission within the forebrain [20]. Glutamate is present in the brain at significant concentrations (as high as $10 \mathrm{mM}$ ) 21], and the progressive labeling of its carbons during metabolism of ${ }^{13} \mathrm{C}$-enriched substrates (commonly glucose) was studied extensively $19,21,22,23,24$. Reduced glutamate neurotransmission in the brain is associated with Alzheimer's disease [24] and with neuropsychiatric disorders [21]. Glycine is normally present at much lower concentrations [19], and its measurement in vivo is therefore challenging. By proton MRS, the single proton signal of glycine was recently resolved in vivo from the overlapping signal of the much more abundant myo-inositol [20, 25]. In vivo $13 \mathrm{C}$ MRS could also measure glycine, which is obtained in neurons from glucose (via serine) [19]. Abnormal levels of glycine are implicated in schizophrenia and other neuropsychiatric disorders 20], and recently glycine was also shown to be a biomarker of tumor malignancy [25].

In this letter, we describe heat-bath cooling of the spin systems of glutamic acid (7 spins: one $\alpha$ proton, ${ }^{13} \mathrm{C} 1$, ${ }^{13} \mathrm{C} 2$, and 4 methylene protons) and glycine (4 spins: two $\alpha$ protons, ${ }^{13} \mathrm{C} 1$, and ${ }^{13} \mathrm{C} 2$ ), depicted in Fig. 11 For these systems, perfect PT from the reset proton(s) can increase $13 \mathrm{C}$ polarization by about 4 -fold [4, 18], and sufficiently high $\mathcal{R}\left(\mathrm{C} 1, \mathrm{H}_{\alpha}\right)$ were expected and confirmed. For each spin system, the entropy was reduced by a single selectivereset (the first two steps of POTENT), and in a different experiment $\mathrm{C} 1$ and $\mathrm{C} 2$ were cooled to a significant extent by adding a PT from the partially repolarized $\alpha$ proton(s) to $\mathrm{C} 2$; step 3 of POTENT. Finally, we examined the possibility of improving the much lower $T_{1}$ ratio, $\mathcal{R}\left(\mathrm{C} 2, \mathrm{H}_{\alpha}\right)$, in order to enable AC, by adding Magnevist ${ }^{\circledR}$, a common Gd-based MRI contrast agent [26].

\section{Materials and Methods}

NMR samples $(0.5 \mathrm{~mL})$ of ${ }^{13} \mathrm{C}$-labeled amino acids $(99 \%$ ${ }^{13} \mathrm{C}$, Cambridge Isotope Laboratories) were prepared in $\mathrm{D}_{2} \mathrm{O}(99.96 \% \mathrm{D})$, and the $\mathrm{pH}$ was adjusted by adding $\mathrm{K}_{3} \mathrm{PO}_{4}$ (anhydrous, 99\% pure, Sigma-Aldrich) and measured using 6.4-8.0 pH sticks (0.2 units, Lyphan); see samples $G$ and $E$ in Table $1, \mathrm{D}_{2} \mathrm{O}$ solutions of Magnevist ${ }^{\circledR}$ were prepared from lyophilized samples of a clinical aqueous solution (0.5M, Bayer HealthCare Pharmaceuticals). A dilute solution of Magnevist in $\mathrm{D}_{2} \mathrm{O}(5 \mathrm{mM})$ was added directly to the NMR tubes with a micropipette in $5 \mu \mathrm{L}$ aliquots, corresponding to incremental Magnevist concentrations of $0.05 \mathrm{mM}$, similar to a recent study of glycine relaxation [27]. Aliquots were added until $T_{1}\left(\mathrm{H}_{\alpha}\right)$ was reduced to about $1 \mathrm{~s}$; see samples $E_{M}$ and $E_{M}^{\prime}$ in Table 1.

All experiments were performed on a Bruker Avance 500 spectrometer with a standard $5 \mathrm{~mm}$ BBO probe and a digital variable temperature control unit, at controlled temperatures $\left(0.1 \mathrm{~K}\right.$ accuracy). $T_{1}$ relaxation times were measured by inversion recovery (with the standard Bruker t1ir pulse sequence), consisting of a prolonged recycle delay $\left(7 T_{1}\right)$, followed by $180^{\circ}, \tau, 90^{\circ}$, and acquisition. 17 logarithmically-spaced $\tau$ values were used, such that a few were below $T_{1}$ and at least one delay exceeded $7 T_{1}$.

The POTENT pulse sequence [1] and its components described above were used with two main modifications; First, the INEPT pulse sequences in steps 1 and 3 were implemented with spin-selective low-power pulses for ${ }^{13} \mathrm{C}$ excitation and inversion, which had shapes of E-BURP1 and I-BURP1 [28], respectively, and a duration of $t=$ $1 \mathrm{~ms}$. Second, during the PT from C2 to C1 in step 1, WALTZ-16 [29] proton decoupling was employed.

Detailed pulse sequences are illustrated in Fig. 2. The HCC relay (Fig. 2a) includes delays for the two INEPTs in step 1 ( $d 4$ and $d 7$ ), and a variable refocusing period, $d 5$, set to be $d 4 / k$ depending on the number, $k$, of $\alpha$ protons [18]; For glutamate, $d 5=1.21 \mathrm{~ms}(d 4=1.71 \mathrm{~ms}, d 7=$ $4.681 \mathrm{~ms})$, while for glycine $d 5=0.39 \mathrm{~ms}(d 4=1.79 \mathrm{~ms}$, $d 7=4.742 \mathrm{~ms}$ ), where $d 5=d 4 / k-t / 2 \mathrm{~ms}$, and the shortening (by $1 / 2 \mathrm{~ms}$ ) compensates for the refocusing I-BURP1 pulse on $\mathrm{C} 1$. The HCC+WAIT pulse sequence (Fig. 2b) employed a long repolarization delay, $d 3 \sim 7 T_{1}\left(\mathrm{H}_{\alpha}\right)$. The delay $d 7$ was optimized (around $1 / 4 J_{C C}$ ) in $0.01 \mathrm{~Hz}$ intervals to maximize the integral of $\mathrm{C} 1$ after HCC+WAIT. For POTENT (Fig. 2r), the first repolarization delay, $d 2 \gtrsim$ $2 T_{1}\left(\mathrm{H}_{\alpha}\right)$, allowed significant cooling of $\mathrm{C} 2$ by the following refocused INEPT. The final delay, $d 3$, was of the order of $T_{2}\left(\mathrm{H}_{\alpha}\right)$ to eliminate small distortions, and not for proton repolarization. Thus, the resulting POTENT is 
truncated. For sample $E_{M}^{\prime}$, in addition to truncated POTENT, we also applied (truncated) $\mathrm{POTENT}^{+}$, where $d 2$ was followed by a $90^{\circ}$ pulse on $\mathrm{C} 2$, resulting in uniform lines [18]. The delay $d 14$ in PT \#2, step 3 of POTENT, was $d 4-t / 2$ (again shortened to compensate for the soft pulse I-BURP1).

A single scan was acquired for the samples in Table 1. In order to obtain the proton spectrum (when cooling the entire spin system), the HCC+WAIT sequence was repeated under the same conditions, while acquiring the proton spectrum (see Fig. 2b). The NMR data were apodized with small $(0.3 \mathrm{~Hz})$ exponential line broadening function prior to Fourier transformation. The spectra were phasecorrected according to the equilibrium spectra, and polarization enhancements were calculated from peak integrals.

\section{Results}

\section{1. $T_{1}$ measurements}

To affirm that ${ }^{13} \mathrm{C}_{2}$-labeled amino acids are suitable for heat-bath cooling, we determined the longitudinal relaxation times (Table1) for glycine, sample $G$, and glutamate, sample $E$, at room temperature $\left(24.0^{\circ} \mathrm{C}\right)$. As expected, $\mathrm{C} 1$ had much slower relaxation, resulting in $T_{1}$ ratios of $\mathcal{R}\left(\mathrm{C} 1, \mathrm{H}_{\alpha}\right) \sim 10$, while $\mathcal{R}\left(\mathrm{C} 2, \mathrm{H}_{\alpha}\right) \sim 1.5$ (see Table 2).

For glycine in $\mathrm{D}_{2} \mathrm{O}$ (sample $G$ ), a long relaxation time was found for the carbonyl, $T_{1}(\mathrm{C} 1)=31.6 \pm 0.5 \mathrm{~s}$, similar to the relaxation time recently reported for an aqueous solution of $1,2{ }^{13} \mathrm{C}_{2}$-glycine $\left(16 \% \mathrm{D}_{2} \mathrm{O}\right), T_{1}(\mathrm{C} 1)^{a q}=$ $27.3 \pm 1.2 \mathrm{~s}$, obtained under similar conditions [27]. For $\mathrm{C} 2$ and the $\alpha$ protons of glycine, we found much more rapid relaxation: $T_{1}(\mathrm{C} 2)=3.75 \pm 0.05 \mathrm{~s}$, in good agreement with the aqueous solution, $T_{1}(\mathrm{C} 2)^{a q}=4.0 \pm 0.2 \mathrm{~s}[27]$ and $T_{1}\left(\mathrm{H}_{\alpha}\right)=2.72 \pm 0.02 \mathrm{~s}$.

For glutamate (sample $E$ ), shorter relaxation times were found, $T_{1}(\mathrm{C} 1)=13.03 \pm 0.04 \mathrm{~s}$, somewhat similar to an aqueous solution, $T_{1}(\mathrm{C} 1)^{a q}=10.2 \pm 0.8 \mathrm{~s}[30], T_{1}(\mathrm{C} 2)=$ $1.96 \pm 0.02 \mathrm{~s}$ and $T_{1}\left(\mathrm{H}_{\alpha}\right)=1.29 \pm 0.02 \mathrm{~s}$. The relaxation times of the methylene protons were similar to $\mathrm{H}_{\alpha}$ (see Table 11).

Addition of Magnevist to glutamate, sample $E_{M}$, decreased all $T_{1}$ relaxation times. Heating glutamate with Magnevist to physiological temperature $\left(37.0^{\circ} \mathrm{C}\right)$, sample $E_{M}^{\prime}$, increased all $T_{1}$ relaxation times by about $40 \%$. In both cases the resulting $T_{1}$ ratios were similar to sample $E$, see Table 2

\subsection{Cooling the spin systems of glycine and glutamate}

Our goal here was to reduce the total entropy of each amino acid spin system, thereby bypassing Shannon's bound (for similar calculations see Ref. [1]). Both spin systems $G$ and $E$ were cooled at room temperature by a single selective-reset, which consisted of an HCC relay that increased the signal of $\mathrm{C} 1$ by a factor of about 3 , followed by a significant delay, $d 3 \sim 7 T_{1}\left(\mathrm{H}_{\alpha}\right)$. The pulse sequence
HCC+WAIT (Fig. 2b) includes two refocused INEPT sequences and employs proton decoupling during the carboncarbon PT, similar to an earlier sequence [31]. C1 retained an enhancement factor of about 1.9 (1.88 \pm 0.01 for glycine, $1.89 \pm 0.01$ for glutamate), such that the total entropy decreased by $\sim 2.6 \varepsilon^{2} / \ln 4$ (resulting from $2.6 \sim 1.9^{2}-1^{2}$ ), where $\varepsilon$ is the equilibrium ${ }^{13} \mathrm{C}$ polarization. The protons repolarized back to equilibrium (within integration error $\lesssim 1 \%$ ) and $\mathrm{C} 2$ returned to $1.00 \varepsilon \pm 0.01 \varepsilon$, see Figure [3, suggesting that elementary heat-bath cooling is sufficient to go beyond the entropy bound. In terms of effective spin temperatures, obtained by taking the quotient of the equilibrium temperature and the cooling factor (as done in Ref [1]), C1 was cooled to $\sim 156 K-158 K$, while the other spins were at the equilibrium temperature of $297.0 \pm 0.1 K$.

For glutamate in the presence of Magnevist (sample $\left.E_{M}\right)$, the HCC+WAIT sequence produced similar cooling as for sample $E$. C1 was cooled by a factor of about 1.9, while the other spins regained their equilibrium polarizations.

\subsection{Heat-bath cooling of $\mathrm{C} 1$ and $\mathrm{C} 2$}

A potentially useful application of heat-bath cooling is to enhance, simultaneously, two labeled carbons by applying the POTENT pulse sequence [1] with no final delay; Fig. 26. For both amino acids (samples $G$ and $E$ ), C1 and $\mathrm{C} 2$ were cooled to a similar extent (about 2.4-fold, see Table 3) when the first delay was $d 2 \sim 2-3 T_{1}\left(\mathrm{H}_{\alpha}\right)$. The very short final delay $(d 3 \lesssim 1 \mathrm{~s})$ was used only to eliminate small distortions. The spin temperatures after cooling were $\sim 120 K-130 K$.

For glutamate with Magnevist (sample $E_{M}$ ), application of steps 1-3 of POTENT resulted in cooling factors of about 2.5 and 2.3 for $\mathrm{C} 1$ and $\mathrm{C} 2$, respectively, similar to sample $E$, reflecting the similarity in $T_{1}$ ratios for both samples (see Table 2).

Application of POTENT to glutamate at physiological temperature of $37.0^{\circ} \mathrm{C}$ ( sample $E_{M}^{\prime}$ ) cooled both carbons by a factor of about 2.5 , quite similar to the cooling obtained at room temperature described above, see bottom part of Table 3. Interestingly, improved cooling at physiological temperature is obtained by $\mathrm{POTENT}^{+}$(Fig. 26), where an additional $90^{\circ}$ pulse was applied to $\mathrm{C} 2$, in order to provide uniform net PT (equal intensity for the lines of the C2 multiplets [18]), see Fig. 4. This sequence allowed a two-fold reduction in the final delay ( $d 3$ of the order of $T_{2}\left(\mathrm{H}_{\alpha}\right)$ ) by transforming erroneous density-matrix elements into non-observable coherences; consequently, for $d 2=2.0 T_{1}\left(\mathrm{H}_{\alpha}\right), \mathrm{C} 1$ and $\mathrm{C} 2$ were cooled by factors of about 2.60 and 2.65 , respectively.

\section{Discussion}

\subsection{Heat-bath cooling of amino acids for in vivo spec- troscopy}

The simultaneous cooling of both labeled carbons at physiological conditions (sample $E_{M}^{\prime}$ ) suggests that heat- 
bath cooling is suitable for 13C-MRS, which detects and quantifies ${ }^{13} \mathrm{C}$-labeled metabolites in vivo, in particular at specific locations within the brain [12]. For glutamate, it was recently shown that the INEPT pulse sequence can be adapted to in vivo 13C-MRS and used for brain spectroscopy with WALTZ decoupling [22, 32]. Heat-bath cooling sequences that combine these elements may therefore be readily adapted to localized in vivo spectroscopy.

Simultaneous cooling of both ${ }^{13} \mathrm{C}$ of 1,2 -labeled amino acids by the POTENT pulse sequence may enhance the detection of such isotopomers in vivo. For glutamate, both $\mathrm{C} 2$-labeled and 1,2-labeled isotopomers are produced in cerebral metabolism of $1,2-{ }^{13} \mathrm{C}_{2}$-glucose [12]. In this case, application of POTENT in vivo is expected to cool C2 in both single and double-labeled isotopomers by means of the final PT (see Fig. 2r). Quantitative analysis may be facilitated by a suitable setting of $d 2$, such that both ${ }^{13} \mathrm{C}$ of the double-labeled isotopomer are cooled to a similar extent, as shown in Table 3 .

Heat-bath cooling of the entire spin system as done here is a preliminary step towards AC, which also demonstrates an important concept (in this case on important biomolecules): On the one hand, reversible polarization compression can cool carbons of the two amino acids further, beyond just reaching the polarization of the sensitive nucleus; On the other hand, heat-bath cooling enables entropy reduction, which is not attainable by polarization compression.

\subsection{Algorithmic cooling of amino acids}

Combining heat-bath cooling and compression provides $\mathrm{AC}$ that can, in theory, cool one or more spins far beyond the entropy bound [2]. In reality, finite $T_{1}$ ratios limit the attainable cooling [10, 33]. On the route to useful AC, one could employ various other amino acid isotopomers, such as 2,3,4- ${ }^{13} \mathrm{C}_{3}$-glutamate observed in vivo 34] or 1,2${ }^{13} \mathrm{C}_{2},{ }^{15} \mathrm{~N}-\mathrm{N}$-acetyl aspartate (NAA). NAA, a major metabolite, is present in the brain at low concentrations and was detected in vivo by 13C-MRS [11, 12, 34]. For this molecule, the amide proton constitutes a second reset spin and the labeled nitrogen (observed in vivo in the brain for glutamate [35]) serves as a computing spin. In both molecules, all computing spins may be significantly enhanced by parallel PT steps (a single reset step is required for NAA). A 3-bit compression step could then cool one of the computing spins beyond the proton polarization. For both molecules, further labeling would enable various practicable cooling algorithms [3, 8, 10]. AC could ideally cool one of the $3(6,7)$ computation spins of fully-labeled glycine (glutamate, NAA), using a single reset spin, by a factor of $4(32,64)$ on top of the PT enhancement $[7,[8]$.

Recently, in vivo 13C-MRS experiments with hyperpolarized biomolecules (e.g., ${ }^{13} \mathrm{C}$-pyruvate) have demonstrated the exciting potential of dissolution DNP and PHIP for ${ }^{13} \mathrm{C}$ spectroscopy and imaging [36, 37]. However, the time scale of metabolic processes within the brain imposes a severe limitation on the effectiveness of ex-vivo hyperpolarization, which is currently limited to few minutes at most [36, 38]. The glutamine-glutamate cycle [23], for example, between neurons and glia cells, is typically followed by $13 \mathrm{C}-\mathrm{MRS}$ over the course of one to several hours [11, 22, 24]. AC of suitably labeled metabolites could be applied at any stage (with no additional equipment) and replenished in vivo, without harming the living tissue. For extended metabolic processes in the brain, a modest cooling (as could be obtained by AC) of around 5-10 fold is feasible in the near future. As a linear relationship between $1 / T_{1}$ and Magnevist concentration was shown for backbone carbons of both amino acids [27, 30], another interesting future avenue is to run $\mathrm{AC}$ with much higher concentration of Magnevist (e.g., $1 \mathrm{mM}$ ).

In solid state NMR, AC was achieved for malonic acid [39, 40], using spin-diffusion (instead of thermalization) as a fast repolarization mechanism for the protons, to enable up to four cycles of AC [40]. Very recently, several cycles of AC were achieved in solution for TCE, using numerically optimized pulses [41]. In the far future, use of electrons as reset spins could enable application of $\mathrm{AC}$ to larger spin systems to obtain highly polarized spins [3, 10].

\section{Conclusion}

Heat-bath cooling of the backbone carbons of glycine and glutamate may be readily applied in vivo, and could also be applicable to other amino acids (and similar metabolites). Going beyond heat-bath cooling, various suitably labeled isotopomers of amino acids and similar metabolites may be cooled effectively by AC (potentially using Magnevist). Heat-bath cooling in the near future, and AC later on, may enhance spectroscopy of slow metabolic processes, particularly in the brain where hyperpolarization is not effective. These methods are safe, require no additional equipment, and may be replenished.

\section{Acknowledgments}

We thank Dr Dorith Goldsher, Dr Yael Balasz and Yosi Atia for enlightening discussions, and we also thank D.G. for a sample of Magnevist. This work is supported in part by the Wolfson Foundation and the Israeli MOD Research and Technology Unit. The work of T.M. was also supported in part by FQRNT through INTRIQ, and by NSERC.

\section{References}

[1] G. Brassard, Y. Elias, J. M. Fernandez, H. Gilboa, J. A. Jones, T. Mor, Y. Weinstein, L. Xiao (2005).

[2] P. O. Boykin, T. Mor, V. Roychowdhury, F. Vatan, R. Vrijen, Proc. Natl. Acad. Sci. USA 99 (2002) 3388.

[3] J. M. Fernandez, S. Lloyd, T. Mor, V. Roychowdhury, Int. J. Quant. Inf. 2 (2004) 461.

[4] O. W. Sørensen, Prog. Nucl. Mag. Res. Spec. 21 (1989) 503.

[5] L. J. Schulman, U. V. Vazirani, in: ACM Symposium on the Theory of Computing (STOC): Proceedings, p. 322. 
[6] T. M. Cover, J. A. Thomas, Elements of Information Theory, Wiley, New York, 1991.

[7] L. J. Schulman, T. Mor, Y. Weinstein, Phys. Rev. Lett. 94 (2005) 120501.

[8] Y. Elias, J. M. Fernandez, T. Mor, Y. Weinstein, Isr. J. Chem. 46 (2006) 371.

[9] L. J. Schulman, T. Mor, Y. Weinstein, SIAM J. Comp. 36 (2007) 1729

[10] Y. Elias, T. Mor, Y. Weinstein, Phys. Rev. A 83 (2011) 042340.

[11] B. Ross, A. Lin, K. Harris, P. Bhattacharya, B. Schweinsburg, NMR Biomed. 16 (2003) 358.

[12] T. B. Rodrigues, S. Cerdán, Concepts Magn. Reson. Part A 27A (2005) 1

[13] J. H. Ardenkjær-Larsen, B. Fridlund, A. Gram, G. Hansson, L. Hansson, M. H. Lerche, R. Servin, M. Thaning, K. Golman, Proc. Natl. Acad. Sci. USA 100 (2003) 10158.

14] P. Bhattacharya, K. Harris, A. P. Lin, M. Mansson, V. A. Norton, W. H. Perman, D. P. Weitekamp, B. D. Ross, MAGMA 18 (2005) 245.

[15] A. M. Oros, N. J. Shah, Phys. Med. Biol. 49 (2004) R105-R153.

[16] J. M. Fernandez, T. Mor, Y. Weinstein, in: Proc. Int. Soc. Opt. Eng. (SPIE), volume 5105, Orlando, Florida, USA, p. 185.

[17] G. A. Morris, R. Freeman, J. Am. Chem. Soc. 101 (1979) 760.

[18] D. P. Burum, R. R. Ernst, J. Mag. Reson. 39 (1980) 163.

[19] E. J. Novotny, R. K. Fulbright, P. L. Pearl, M. K. Gibson, D. L. Rothman, Annals of Neurology 54 (2003) S25.

[20] A. P. Prescot, B. B. Frederick, L. Wang, J. Brown, J. E. Jensen, M. J. Kaufman, P. F. Renshaw, Magn. Reson. Med. 55 (2006) 681.

[21] N. Sailasuta, L. W. Robertson, K. C. Harris, A. L. Gropman, P. S. Allen, B. D. Ross, J. Mag. Reson. 195 (2008) 219.

[22] R. Gruetter, G. Adriany, I.-Y. Choi, P.-G. Henry, H. Lei, G. Öz, B. Ross, A. Lin, K. Harris, P. Bhattacharya, B. Schweinsburg, NMR Biomed. 16 (2003) 313.

[23] D. L. Rothman, K. L. Behar, F. Hyder, R. G. Shulman, Annu. Rev. Physiol. 65 (2003) 41.

[24] A. Lin, F. Shic, C. Enriquez, B. Ross, MAGMA 16 (2003) 29

[25] C. Choi, D. Douglas, H. Hawesa, A. Jindal, C. Storey, I. Dimitrov, Magn. Reson. Med. 62 (2009) 1305.

[26] S. Laurent, L. V. Elst, R. N. Muller, Contrast Media Mol. Imaging 1 (2006) 128 .

[27] C. Gabellieri, M. O. Leach, T. R. Eykyn, Contrast Media Mol Imaging 4 (2009) 143.

[28] H. Geen, R. Freedman, J. Mag. Reson. 93 (1991) 93.

[29] A. J. Shaka, J. Reeler, T. Frenkiel, R. Freeman, J. Mag. Reson. 52 (1983) 335.

[30] R. B. van Heeswijk, F. D. Laus, S. Morgenthaler, R. Gruetter Magn. Resno. Imaging. 25 (2007) 821.

[31] L. Poppe, S. Gröger, F. Zablocki, Magn. Reson. Chem. 37 (1999) 382.

[32] S. Xu, J. Shen, J. Mag. Reson. 182 (2006) 221.

[33] G. Brassard, Y. Elias, T. Mor, Y. Weinstein, 2012. Manuscript in preparation.

[34] D. K. Deelchand, K. Uğrbil, P.-G. Henry, Magn. Reson. Med. 55 (2006) 279

[35] K. Kanamori, B. D. Ross, Magn. Reson. Med. 41 (1999) 456.

[36] K. Golman, S. J. Petersson, Acad. Radiol. 13 (2006) 932.

[37] J. Kurhanewicz, R. Bok, S. J. Nelson, D. B. Vigneron, J. Nucl. Medicine 49 (2008) 341.

[38] B. Ross, P. Bhattacharya, S. Wagner, T. Tran, N. Sailasuta, Am. J. Neuroradiol. 31 (2010) 24

[39] J. Baugh, O. Moussa, C. A. Ryan, A. Nayak, R. Laflamme, Nature 438 (2005) 470

[40] C. A. Ryan, O. Moussa, J. Baugh, R. Laflamme, Phys. Rev. Lett. 100 (2008) 140501.

[41] Y. Atia, Y. Elias, T. Mor, Y. Weinstein, 2011. Manuscript in preparation.

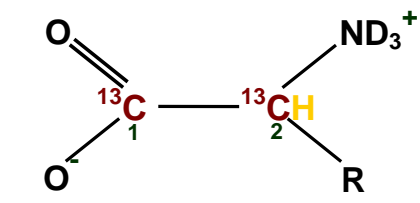

$\mathbf{R}=\left\{\begin{array}{l}\mathrm{H} \text { (glycine) } \\ \mathrm{CH}_{2} \mathrm{CH}_{2} \mathrm{COOD} \text { (glutamic acid) }\end{array}\right.$

Figure 1: Amino acids used in heat-bath cooling experiments.

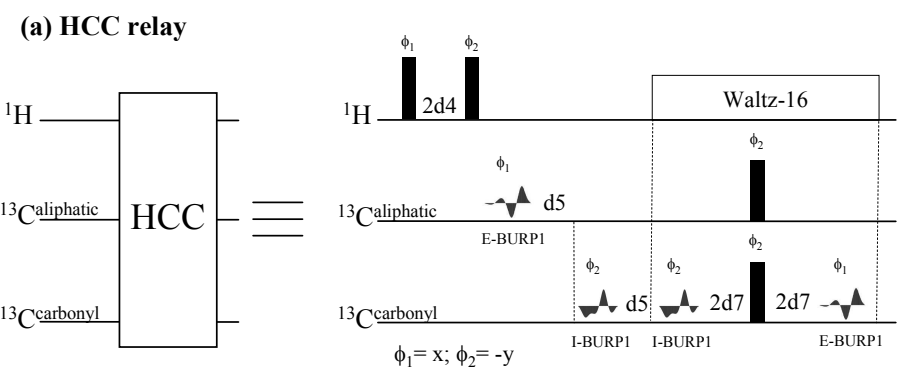

(b) HCC+WAIT
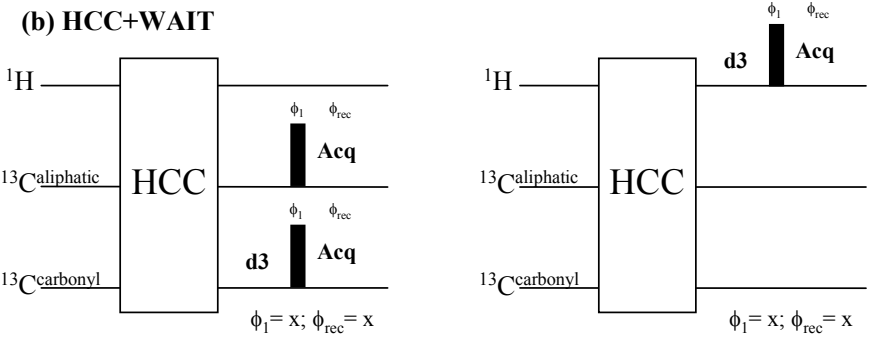

(c) POTENT / POTENT $^{+}$

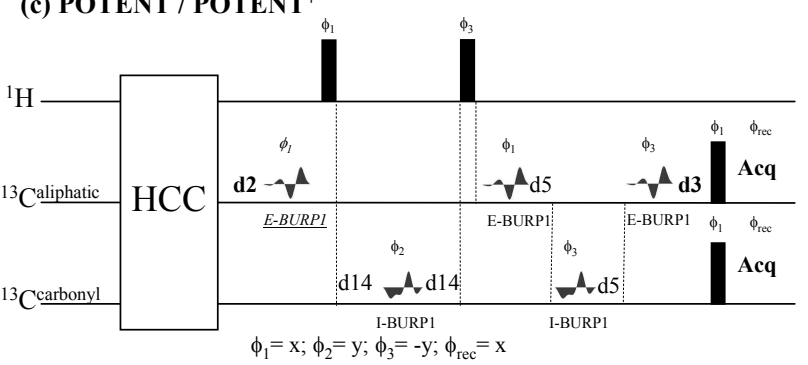

Figure 2: Pulse-sequences for heat-bath cooling of amino acids. Narrow black bars denote $90^{\circ}$ non-selective pulses, while E-BURP1 and I-BURP1 represent $1 \mathrm{~ms}$ spin-selective BURP pulses [28]. (a) The HCC relay was implemented by two refocused INEPT sequences. (b) A significant delay $\left(d 3 \sim 7 T_{1}\left(\mathrm{H}_{\alpha}\right)\right.$ ) following the relay restored the polarization of all protons. (c) Alternatively, both carbons were cooled by truncated POTENT sequences, where $\mathrm{POTENT}^{+}$includes an additional E-BURP1 $90^{\circ}$ pulse (highlighted) on $\mathrm{C} 2$ after $d 2$. Acquisition (after $90^{\circ}$ pulse) is performed for both nuclei (b) or only for ${ }^{13} \mathrm{C}$ (c). In figure (c) we denote the pulse sequences as POTENT/POTENT ${ }^{+}$because for long $d 3$ the proton would repolarize (step 4 of POTENT). 

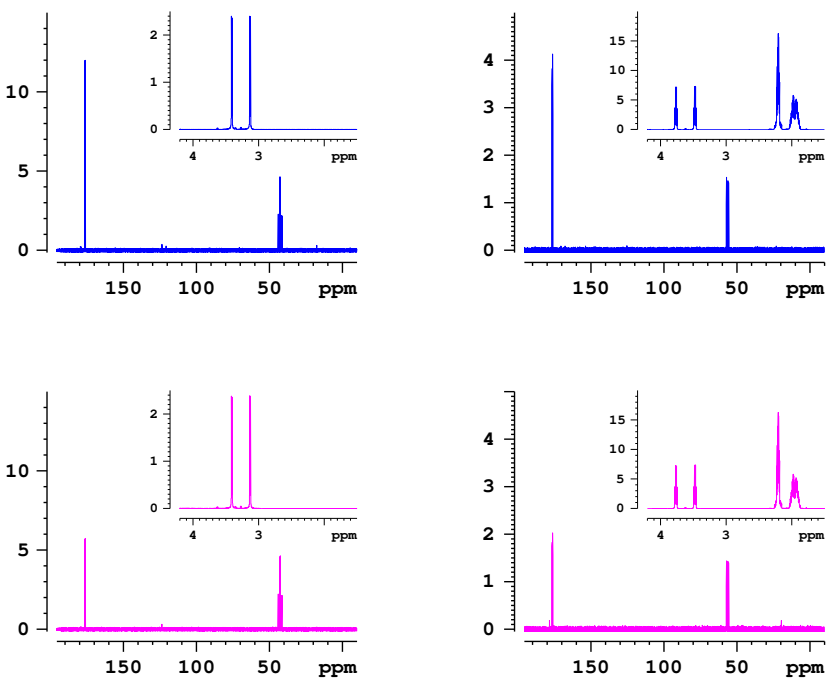

Figure 3: Cooling beyond Shannon's bound. ${ }^{13} \mathrm{C}$ spectra (proton spectra in insets) of glycine (left, sample $G$ ) and glutamate (right, sample $E$ ) before (bottom) and after (top) the HCC+WAIT experiment. C1 was cooled by a factor of $\sim 1.9$, while the protons fully repolarized and $\mathrm{C} 2$ regained its equilibrium polarization.

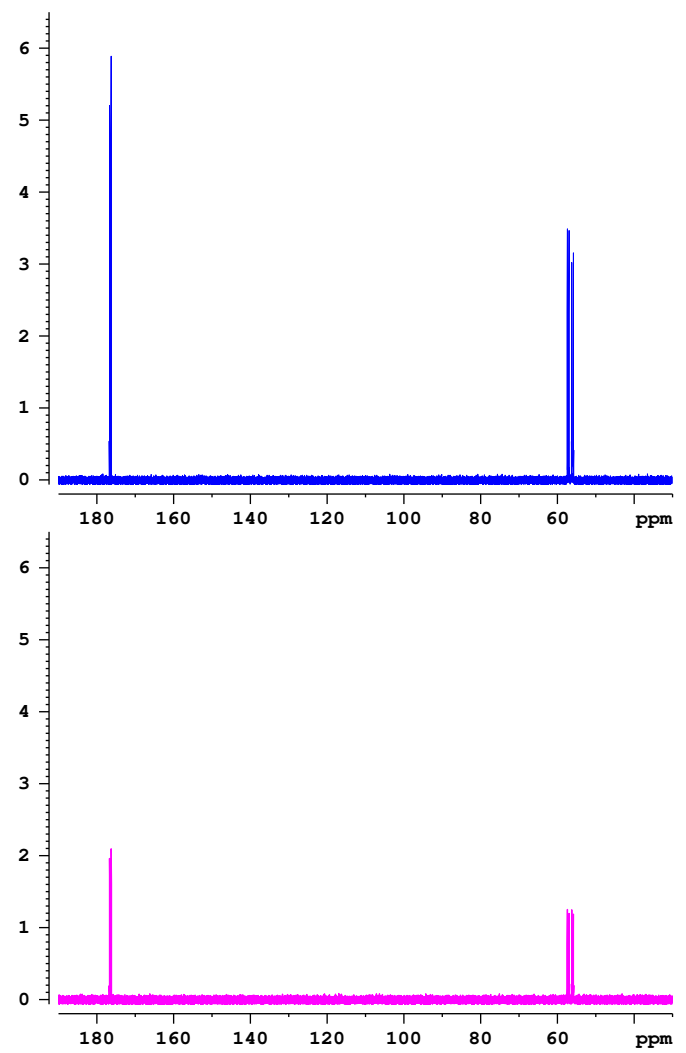

Figure 4: Simultaneous cooling of both backbone carbons of glutamate at physiological temperature with Magnevist (sample $E_{M}^{\prime}$ ) using $\mathrm{POTENT}^{+}$, see Table 3 for details. Spectra are at equilibrium (bottom) and after cooling (top). 
Table 1: $T_{1}$ values (in seconds) of ${ }^{13} \mathrm{C}$-labeled glycine and glutamate in $\mathrm{D}_{2} \mathrm{O}(\sim 300 \mathrm{mM})$. Samples $E_{M}$ and $E_{M}^{\prime}$ contain $0.05 \mathrm{mM}$ Magnevist. Errors are standard deviations of 3-10 single scans.

\begin{tabular}{clllcccc}
\hline sample & Amino acid & $\mathrm{T}[\mathrm{K}]$ & $T_{1}(\mathrm{C} 1)$ & $T_{1}(\mathrm{C} 2)$ & $T_{1}\left(\mathrm{H}_{\alpha}\right)$ & $T_{1}(\mathrm{H} 3)$ & $T_{1}(\mathrm{H} 4)$ \\
\hline$G$ & glycine & 297 & $31.6 \pm 0.5$ & $3.75 \pm 0.05$ & $2.72 \pm 0.02$ & & \\
$E$ & glutamate & 297 & $13.03 \pm 0.04$ & $1.96 \pm 0.02$ & $1.29 \pm 0.02$ & $1.001 \pm 0.006$ & $1.281 \pm 0.003$ \\
$E_{M}$ & glutamate & 297 & $10.2 \pm 0.1 \mathrm{~b}$ & $1.84 \pm 0.02$ & $1.10 \pm 0.01$ & $0.920 \pm 0.003$ & $1.160 \pm 0.001$ \\
$E_{M}^{\prime}$ & glutamate & 310 & $14.36 \pm 0.06$ & $2.66 \pm 0.04$ & $1.50 \pm 0.01$ & $1.270 \pm 0.004$ & $1.606 \pm 0.004$
\end{tabular}

${ }^{\mathrm{a}}$ Molar ratios of $\mathrm{K}_{3} \mathrm{PO}_{4}$ to amino acid were 0.5 for glycine ( $\mathrm{pH} 8$ ) and 0.8 for glutamate (pH 7.5).

${ }^{\mathrm{b}}$ For sample $E_{M}$, only two measurements were taken for C1.

Table 2: $T_{1}$ ratios for the samples in Table 1

\begin{tabular}{lccc}
\hline sample & Magnevist $[\mathrm{mM}]$ & $\mathcal{R}\left(\mathrm{C} 1, \mathrm{H}_{\alpha}\right)$ & $\mathcal{R}\left(\mathrm{C} 2, \mathrm{H}_{\alpha}\right)$ \\
\hline$G$ & 0 & $11.6 \pm 0.3$ & $1.38 \pm 0.03$ \\
$E$ & 0 & $10.1 \pm 0.2$ & $1.52 \pm 0.04$ \\
$E_{M}$ & 0.05 & $9.3 \pm 0.2$ & $1.67 \pm 0.03$ \\
$E_{M}^{\prime}$ & 0.05 & $9.6 \pm 0.1$ & $1.77 \pm 0.04$
\end{tabular}

Table 3: Simultaneous cooling of both backbone carbons by POTENT without (top two rows) and with added Magnevist. In the last row, $\mathrm{POTENT}^{+}$was used (see Fig. 2 2 and text). Results are averages and standard deviations of at least 3 single-scan measurements.

\begin{tabular}{clccc}
\hline sample & $d 2 / T_{1}\left(\mathrm{H}_{\alpha}\right)^{\mathrm{a}}$ & $d 3[\mathrm{~s}]^{\mathrm{a}}$ & C1 factor ${ }^{\mathrm{b}}$ & C2 factor $^{\mathrm{b}}$ \\
\hline$G$ & 2.2 & 1.0 & $2.32 \pm 0.06$ & $2.52 \pm 0.09$ \\
$E^{2}$ & 3.1 & 1.0 & $2.45 \pm 0.01$ & $2.29 \pm 0.02$ \\
\hline$E_{M}$ & $1.8-2.7$ & 1.0 & $2.45 \pm 0.08$ & $2.29 \pm 0.04$ \\
$E_{M}^{\prime}$ & 2.7 & 1.0 & $2.51 \pm 0.04$ & $2.49 \pm 0.04$ \\
$E_{M}^{\prime}$ & $2.0{ }^{\mathrm{c}}$ & 0.5 & $2.61 \pm 0.01$ & $2.65 \pm 0.01$
\end{tabular}

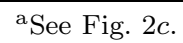

${ }^{\mathrm{b}}$ The ratio between signals with and without cooling.

${ }^{\mathrm{c}}$ With the delay used for POTENT $(d 2=2.7 \mathrm{~s})$, POTENT $^{+}$ cooled $\mathrm{C} 1$ and $\mathrm{C} 2$ by respective factors of about 2.5 and 2.7 .
} 